\title{
Letter to the Editor: Use of some inappropriate terms in Spanish in Oral Medicine and Pathology
}

\author{
José-Manuel Aguirre-Urizar ${ }^{1}$, Adalberto Mosqueda-Taylor ${ }^{2}$ \\ ${ }^{1}$ Departamento de Estomatología II. UFI 11/25. Universidad del País Vasco/EHU. Leioa. España \\ ${ }^{2}$ Departamento de Atención a la Salud. Universidad Autónoma Metropolitana. Xochimilco. México D.F. México
}

\author{
Correspondence: \\ Unidad de Patología Oral y Maxilofacial Servicio \\ Clínica Odontológica Facultad de Medicina y \\ Odontología Universidad del País Vasco \\ EHU Leioa 48940. Vizcaya \\ España. (Spain) \\ josemanuel.aguirre@ehu.eus
}

Received: 10/01/2016 Accepted: 14/01/2016

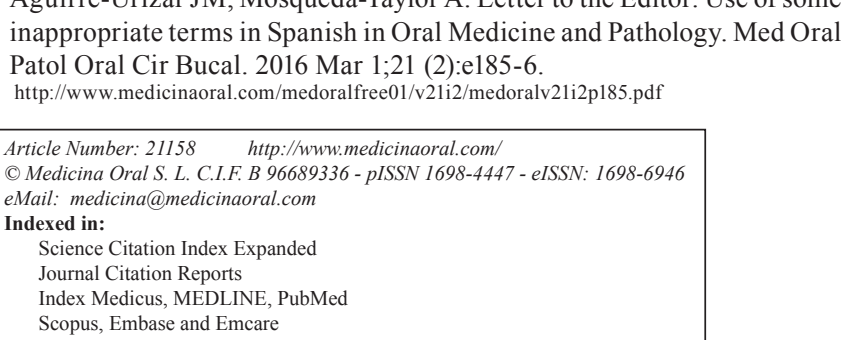
inappropriate terms in Spanish in Oral Medicine and Pathology. Med Oral Patol Oral Cir Bucal. 2016 Mar 1;21 (2):e185-6.

http://www.medicinaoral.com/medoralfree01/v21i2/medoralv21i2p185.pdf 
mild impairment of health. The word "trastornar" has, among others, the meanings: 1) reverse the regular order of something and 2) to alter the normal functioning of something or the activity of someone.

Our proposal is to translate "oral potentially malignant disorders" into Spanish as "trastornos orales potencialmente malignos".

Similarly, the term "verrucous", which defines a similar clinical appearance of verrucae is very often mistakenly translated into Spanish as "verrucoso", a word that has no basis in the DLERAE (1). We therefore believe that this word should be translated as "verrugoso", and therefore "verrucous leukoplakia" should be translated as "leucoplasia verrugosa", analogous to the translation of "verruga" for the English term "verruca".

Finally, another inadequate translation into Spanish occurs with the word "necrotizing", which is usually translated incorrectly as "necrotizante", a term that does not exist in the DLERAE (1). Thus, we believe it should be translated into Spanish as "necrosante" coming from the verb "necrosar" (action to produce necrosis)1, so that "necrotizing sialometaplasia" would be translated as "sialometaplasia necrosante" as "necrotizing granulomas" would be "granulomas necrosantes". The confusion stems from the act of trying to translate the English verbs ending with "tize" as "tizar", when these should be left in their Spanish infinitive form "ar", and therefore "anesthetize" is "anestesiar" or "phagocytize" is "fagocitar", etc.

\section{References}

1. Diccionario de la Lengua Española de la Real Academia Española. http://dle.rae.es/?w=diccionario. RAE. España. October 2014. 